\title{
Role of Functional Genetic Variation in the Dopamine D2 Receptor (DRD2) in Response to Bupropion and Nicotine Replacement Therapy for Tobacco Dependence: Results of Two Randomized Clinical Trials
}

\author{
Caryn Lerman*,', Christopher Jepson', E Paul Wileyto', Leonard H Epstein², Margaret Rukstalis', \\ Freda Patterson', Vyga Kaufmann', Stephanie Restine ${ }^{3}$, Larry Hawk ${ }^{4}$, Ray Niaura ${ }^{5}$ and Wade Berrettini ${ }^{6}$ \\ 'Department of Psychiatry and Abramson Cancer Center, Tobacco Use Research Center, University of Pennsylvania, Philadelphia, PA, USA; \\ ${ }^{2}$ Department of Pediatrics, State University of New York (SUNY) Buffalo, Buffalo, NY, USA; ${ }^{3}$ Molecular Diagnosis and Genotyping Facility, \\ Abramson Cancer Center, University of Pennsylvania, Philadelphia, PA, USA; ${ }^{4}$ Department of Psychology, State University of New York (SUNY) \\ at Buffalo, Buffalo, NY, USA; ${ }^{5}$ Department of Psychiatry \& Human Behavior, Transdisciplinary Research, Butler Hospital, Providence, RI, USA; \\ ${ }^{6}$ Department of Psychiatry, Center for Neurobiology and Behavior, University of Pennsylvania, Philadelphia, PA, USA
}

\begin{abstract}
Although bupropion and nicotine replacement therapy (NRT) are efficacious tobacco dependence treatments, there is substantial interindividual variability in therapeutic response and most smokers relapse. Pharmacogenetics research may improve treatment outcomes by identifying genetic variants predictive of therapeutic response. We investigated the roles of two functional genetic variants in the dopamine D2 receptor (DRD2) gene in response to pharmacotherapy for tobacco dependence among participants in two randomized clinical trials with a 6-month follow-up period: a double-blind placebo-controlled trial of bupropion $(n=4 \mid 4)$ and an open label trial of transdermal nicotine vs nicotine nasal spray $(n=368)$. At the end of the treatment phase, a statistically significant $(p=0.0 \mathrm{l})$ interaction between the DRD2 - I4IC Ins/Del genotype and treatment indicated a more favorable response to bupropion among smokers homozygous for the Ins $C$ allele compared to those carrying a Del $C$ allele. By contrast, smokers carrying the Del $C$ allele had statistically significantly $(p=0.006)$ higher quit rates on NRT compared to those homozygous for the Ins C allele, independent of NRT type. The C957T variant was also associated $(p=0.03)$ with abstinence following NRT. These results suggest that bupropion may be the preferred pharmacologic treatment for smokers homozygous for the DRD2 - I4I Ins C allele, while NRT may be more beneficial for those who carry the Del C allele. Study findings require confirmation in additional larger samples before they are applied in practice. Neuropsychopharmacology (2006) 3 I, 23 I-242. doi:I 0. I038/sj.npp. I 30086I; published online I0 August 2005
\end{abstract}

Keywords: bupropion; nicotine; genetics; dopamine; smoking; treatment

\section{INTRODUCTION}

Currently, there are two FDA-approved pharmacotherapies for tobacco dependence, nicotine replacement therapy (NRT) and bupropion. Although both treatments have proven efficacy relative to placebo, there is substantial interindividual variability in therapeutic response and few smokers are able to maintain long-term abstinence (Dale

\footnotetext{
*Correspondence: Dr C Lerman, Department of Psychiatry and Abramson Cancer Center, Tobacco Use Research Center, University of Pennsylvania, 3535 Market Street, Suite 4100, Philadelphia, PA 19104, USA, Tel: + I 215746 7I4I, Fax: + I 215746 7I40,

E-mail: clerman@mail.med.upenn.edu

Received 12 November 2004; revised 9 June 2005; accepted 27 June 2005

Online publication: 7 July 2005 at http://www.acnp.org/citations/ Npp070705040532/default.pdf
}

et al, 2001; Fiore et al, 1994; Hurt et al, 1997; Jorenby et al, 1999; Transdermal Nicotine Study Group, 1991). Emerging research in pharmacogenetics (Evans and Relling, 2004; Roses, 2000) may improve the outcomes of tobacco dependence treatment by allowing practitioners to individualize pharmacotherapy based on smokers' genotypes (Lerman and Niaura, 2002; Lerman et al, 2005).

Genetic variation in the dopamine pathway is a plausible target for pharmacogenetic studies of tobacco dependence and treatment response. There is abundant empirical support for the role of dopamine in the rewarding effects of nicotine (Balfour, 2004; Di Chiara and Imperato, 1988; Pontieri et al, 1996). Further, inhibition of dopamine reuptake is one putative mechanism for the beneficial effects of bupropion (Ascher et al, 1995; Sanchez and Hyttel, 1999). Consistent with evidence for the importance of dopamine in nicotine dependence, the dopamine D2 
receptor (DRD2) Taq1 A1 variant is associated with an increased likelihood of nicotine abstinence symptoms (Gilbert et al, 2004), a reduced likelihood of sustained abstinence (Cinciripini et al, 2004), and greater efficacy of transdermal nicotine (TN) treatment relative to placebo (Johnstone et al, 2004; Yudkin et al, 2004). While initial findings relating the DRD2 Taq1 polymorphism to therapeutic response are promising, evidence for an association with smoking status in case-control studies has not been consistent (Bierut et al, 2000; Spitz et al, 1998). The functional significance of genetic variation at the Taq1 locus has not been fully elucidated; however, the A1 allele correlates with altered receptor density and binding characteristics (Noble et al, 1991; Ritchie and Noble, 1996; Thompson et al, 1997). Furthermore, recent data indicate that the Taq1 locus is actually $10 \mathrm{~kb} 3^{\prime}$ to the end of the $D R D 2$ gene in a kinase gene of unknown function (ANKK1) (Neville et al, 2004).

In an effort to elucidate the functional consequences of genetic variation in $D R D 2$, additional sequencing has identified single-nucleotide polymorphisms (SNPs) that may influence DRD2 receptor expression by altering transcription or translation. An insertion/deletion variant in the DRD2 promoter region (DRD2 - 141C Ins/Del) has been identified, with increased transcriptional efficiency observed with the more common -141C Ins allele as compared to the -141C Del allele (Arinami et al, 1997). In addition, Duan et al (2003) recently identified a functional synonymous SNP in DRD2 (C957T) that decreases mRNA stability and protein synthesis. While previous studies have reported associations of the $-141 \mathrm{C}$ Ins/Del variant with schizophrenia (Arinami et al, 1997; Breen et al, 1999), no studies have examined associations of the $-141 \mathrm{C}$ Ins/Del or C957T SNPs with smoking behavior or response to pharmacotherapy.

We investigated the roles of the $-141 \mathrm{C}$ Ins/Del and C957T $D R D 2$ variants in response to pharmacotherapy for tobacco dependence in two independent randomized trials. The first of these was a double-blind placebo-controlled trial of bupropion (Lerman et al, 2002, 2003), and the second was an open-label trial comparing TN to nicotine nasal spray (NS) (Lerman et al, 2004a, b).

\section{MATERIALS AND METHODS}

\section{Participants and Procedures (Study 1, Bupropion)}

Smokers responding to local media advertisements for free smoking cessation treatment and to physician referrals were screened for eligibility and recruited from April 1999 to October 2001 at Georgetown University (Washington, DC) and SUNY Buffalo (New York). The institutional review boards from both universities approved the research protocols. Figure 1 shows the flow of participants through the enrollment, treatment, and follow-up phases of the trial. Participants who were randomized, but did not enter treatment (ie those not included in the intent-to-treat analysis), did not differ significantly from those included in the outcome analysis in terms of demographics, smoking rate, nicotine dependence, or DRD2 - 141C genotype (all $p$-values >0.10) (genotyping for DRD2 C957T was not performed for participants not entering the trial).
Participants in the final analysis included 414 smokers who were $\geqslant 18$ years of age, reported smoking $\geqslant 10$ cigarettes a day for the prior 12 months, and provided informed consent for both genotyping and treatment. Exclusion criteria included pregnancy, a history of DSMIV axis I psychiatric disorder, seizure disorder, and current use of antidepressants or other psychotropic medications. Analyses were limited to persons of European ancestry in order to reduce bias due to racial admixture.

The study was a double-blind randomized clinical trial of bupropion $v s$ placebo for smoking cessation. Randomization was determined by a computer-generated randomization scheme operated by a senior data manager; stratification was carried out by study site. Allocation was concealed from the counselors who delivered the interventions and from the study assistants who assessed the outcomes.

Participants at both sites received identical assessments and treatments. At an initial visit to the smoking clinic, participants provided a $40-\mathrm{ml}$ blood sample and completed a set of standardized self-report questionnaires. All participants received 10 weeks of either placebo or bupropion. Bupropion treatment was delivered according to the standard therapeutic dose $(150 \mathrm{mg} /$ day for the first 3 days, followed by $300 \mathrm{mg} /$ day). All participants also received seven sessions of standardized behavioral group counseling, focusing on self-monitoring and behavioral modification approaches. All participants were instructed to quit smoking on a target quit date (TQD) 2 weeks after initiating medication and counseling.

Smoking status was assessed by telephone interview at the end of treatment (EOT) (8 weeks post-TQD) and at 6 months after the TQD using a validated timeline followback method (Brown et al, 1998). Interviewers were blind to study group assignment. Participants who reported complete abstinence (not even a puff of a cigarette) for at least the 7 days prior to the assessment were asked to complete an in-person visit for biochemical verification of abstinence. Saliva cotinine testing was performed for participants who reported abstinence at a given time-point using a gas-liquid chromatography method (Feyerabend and Russell, 1990). Of 175 participants self-reporting abstinence at EOT, 146 $(83 \%)$ provided a sample for cotinine verification; of 125 participants reporting abstinence at 6-months, $108(86 \%)$ provided a sample.

Adverse events were self-reported on a weekly basis using a standardized measure of severity of potential side effects. Any severe or persistent side effects were reviewed with the study physician.

\section{Participants and Procedures (Study 2, NRT)}

Participants were recruited at Georgetown University (Washington, DC) and the University of Pennsylvania (Philadelphia, PA), from February 2000 to August 2003, using methods identical to those described above. The institutional review boards from both universities approved the research protocols. Figure 2 shows the flow of participants through the enrollment, treatment, and follow-up phases of the trial. Subjects who were randomized, but did not enter treatment (ie those not included in the intent-to-treat analysis), did not differ significantly from 


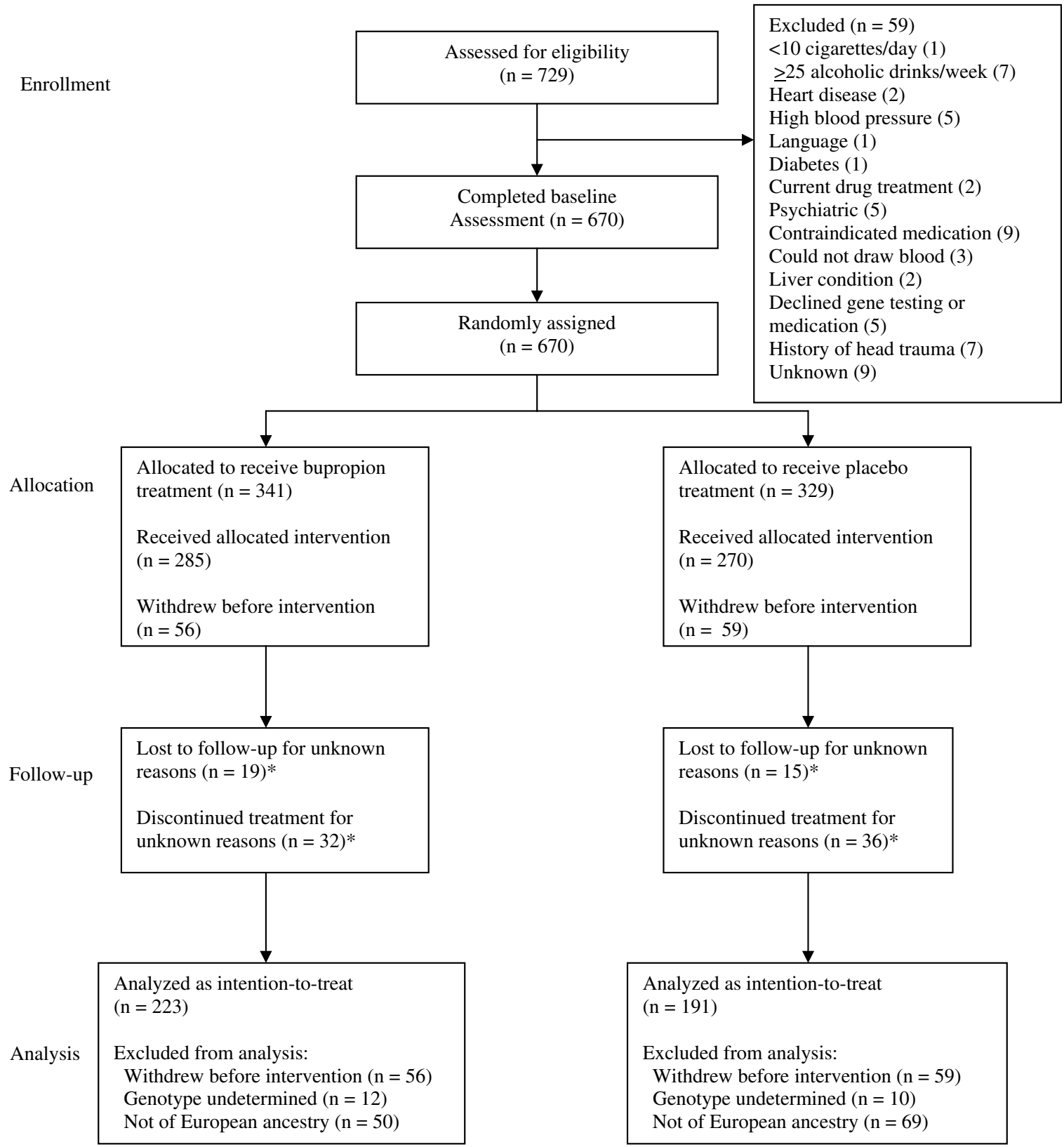

*Included in intent-to-treat analysis

Figure I Flow diagram of participation for Bupropion Trial (Study I).

those included in the outcome analysis in terms of demographics, smoking rate, or nicotine dependence (all $p$-values $>0.20$ ). There was a marginal association of $-141 \mathrm{C}$ Ins/Del genotype with entry into treatment $(p=0.07)$.

Participants were 368 current cigarette smokers of European ancestry who were ages $\geqslant 18$ years, smoked at least 10 cigarettes per day for the prior 12 months, and provided informed consent for both genotyping and treatment. Exclusion criteria included: pregnancy or lactation, uncontrolled hypertension, unstable angina, heart attack or stroke within the past 6 months, current treatment or recent diagnosis of cancer, drug or alcohol dependence, current diagnosis or history of a DSM-IV axis I psychiatric disorder, and current use of bupropion or nicotinecontaining products other than cigarettes.

The trial was an open-label randomized clinical trial of two forms of NRT for smoking cessation: TN $v s$ NS. Randomization was performed as described above for Study 1. Allocation to treatment could not be concealed from the counselors or study assistants who delivered the medication to participants. NS (Nicotrol ${ }^{\mathbb{R}}$; Pharmacia, Helsingborg, Sweden) was initiated on the morning of the target quite date (TQD) (after 2 weeks of counseling) and provided to participants over an 8-week period according to the standard regimen. TN (Nicoderm ${ }^{\circledR}$ CQ; Glaxo Smith Kline, Research Triangle Park, NC) was used by participants over an 8-week treatment period, beginning with the morning of 




Figure 2 Flow diagram of participation for NRT Trial (Study 2).

the TQD, according to the standard regimen. All participants received seven sessions of standardized behavioral group counseling and adverse events were reported as described above for Study 1.

Self-report data on smoking status were obtained at EOT and 6-month follow-up, as described above for Study 1. Participants who reported complete abstinence (not even a puff of a cigarette) for at least the 7 days prior to the assessment were asked to complete an in-person visit for biochemical verification of abstinence using exhaled carbon monoxide (CO) reading (cotinine verification was not used because the treatments contained nicotine). Of 178 participants self-reporting abstinence at EOT, $136(76 \%)$ provided a CO sample for verification; of 115 participants reporting abstinence at 6-months, $83(72 \%)$ provided a CO sample.

\section{Measures (Studies 1 and 2)}

Genotype. Genotyping for the DRD2 -141C Ins/Del and C957T variants was completed using the ABI Prism ${ }^{\circledR}$ 7900HT Sequence Detection System (Applied Biosystems, Foster City, CA). PCR was performed with $2.25 \mathrm{ng}$ of DNA, $2.5 \mu \mathrm{l}$ of ABI Taqman ${ }^{\circledR}$ Universal Mastermix (Foster City, CA), $0.125 \mu$ l of water, and $0.125 \mu \mathrm{l}$ of 40X Assay by Design SNP Assay for the DRD2 variants (ABI, Foster City, CA). The $5 \mu \mathrm{l}$ reactions were performed in a 384-well plate (ABI, 
Foster City, CA). The plates were thermal cycled using the following conditions: (1) $2 \mathrm{~min}$ hold at $50^{\circ} \mathrm{C}$ to activate the AmpErase ${ }^{\circledR}$ Uracil $N$-glycosylase (UNG), an enzyme in the Universal Mastermix used to prevent PCR contamination; (2) $10 \mathrm{~min}$ denaturation step at $95^{\circ} \mathrm{C}$; (3) 50 cycles of $95^{\circ} \mathrm{C}$ for $15 \mathrm{~s}$ and $60^{\circ} \mathrm{C}$ for $1 \mathrm{~min}$; (4) $4^{\circ} \mathrm{C}$ hold forever. The plates were scanned utilizing the Allelic Discrimination End-Point Analysis on the ABI Prism ${ }^{\circledR} 7900$ HT Sequence Detection System. The Allelic Discrimination data were analyzed by the AutoCall algorithm of the SDS v2.1 Software (ABI, Foster City, CA). The DRD2 assays were validated by genotyping 40 samples from both the Coriell Caucasian Human Variation Panel of 50 (HD50CAU) and the Coriell African American Human Variation Panel of 50 (HD50AA) along with 15 samples from the Coriell CEPH/UTAH Pedigree 1333. The Human Variation Panel samples yielded high-quality PCR amplification and genotypes, which could be called with certainty by the ABI software for all 95 samples. The CEPH family genotypes were analyzed using PedCheck (http://watson.hgen.pitt.edu/register/soft_ doc.html) and no Mendelian inheritance errors were found.

For the $-141 \mathrm{C}$ Ins/Del variant, participants were classified based on the presence or absence of the $\mathrm{N}$ null $(\mathrm{Del})$ allele (CC vs $\mathrm{CN}$ or $\mathrm{NN}$ ); because of the very small number of $\mathrm{NN}$ cases in the analysis, we combined $\mathrm{CN}$ and $\mathrm{NN}$ into a single category. For the C957T variant, participants were classified as CC, CT, or TT.

Covariates. Sex, education, age, and smoking rate were assessed at baseline. Nicotine dependence was also assessed at baseline using the Fagerström Test for Nicotine Dependence (Heatherton et al, 1991). The Center for Epidemiological Studies Depression (CES-D) measure (Radloff, 1977) was used to assess baseline depression symptoms.

Primary outcome. In accordance with recommendations of the SRNT expert panel (Hughes et al, 2003; SRNT Subcommittee on Biochemical Verification, 2002), participants were classified as abstinent if they self-reported abstinence (not even a puff) for each of the 7 days immediately prior to the follow-up point (ie point-prevalence), and provided a cotinine reading of $\leqslant 15 \mathrm{ng} / \mathrm{ml}$ (Study 1 ) or a $\mathrm{CO}$ reading of $\leqslant 10 \mathrm{ppm}$ (Study 2). Consistent with these recommendations, we presumed that those who failed to complete the follow-up, or failed to provide a biosample for biochemical verification, had relapsed.

Intermediate outcomes. A self-report measure of withdrawal symptoms assessed the severity (in the past 7 days) of common symptoms, including items such as irritability, difficulty concentrating, anxious/tense, insomnia, drowsiness, nausea, and general physical complaints (eg sweating, dizziness) (Hughes et al, 1984; Piasecki et al, 2000). Responses to items (from $0=$ not at all to $3=$ severe) were summed to create a withdrawal severity index. In the bupropion trial, this instrument was administered at baseline (approximately 3 weeks prior to the TQD), and again at 1 week pre-TQD, on the TQD, and at 1,2 , and 3 weeks post-TQD; in the NRT trial, withdrawal symptoms were assessed at baseline (approximately 3 weeks prior to the TQD), and again at 1 week pre-TQD, on the TQD, and at 1 and 2 weeks post-TQD. A side effects checklist was used for each study to assess the severity of physical complaints potentially associated with treatment (17 items for the bupropion trial and 17 items for the NRT trial). Responses to items (from $0=$ none to $3=$ severe) were summed to create a side effects severity index. In the bupropion trial, this instrument was administered at 1-week pre-TQD, on the TQD, and at 1, 2, 3, and 5 weeks post-TQD; in the NRT trial, side effects were assessed on the TQD and again at 1, 2, 4, and 6 weeks post-TQD. Depression symptoms were reassessed at EOT and at 6-month follow-up using the CES$\mathrm{D}$ as described above.

Compliance in the bupropion trial was assessed based on a standard pill count measure. In the NRT trial, participants assigned to TN recorded their daily application of patches, and those assigned to NS recorded the number of doses per day. Because use of NRT may be confounded with smoking status (ie participants may discontinue NRT if they relapse), we focused on average use of TN or NS during the first 2 weeks of treatment.

\section{Statistical Analysis (Studies 1 and 2)}

We calculated that a sample size of at least 165 participants per group was needed to detect an odds ratio of 4.7 for a genotype by treatment interaction with power $\geqslant 0.80$ (Power and Statistical Analysis Software, NCSS, Kaysville, UT). All analyses were intent-to-treat. Bivariate associations of genotypes and treatment group with abstinence were assessed using $\chi^{2}$ tests. Logistic regression models of abstinence at EOT and 6-month follow-up were then estimated. Predictors included the DRD2 - 141C Ins/Del and DRD2 C957T genotypes, sex, nicotine dependence, treatment group, and interactions of each genotype with treatment group. Both genotypes were treated as dichotomous (0/1) variables, with codes of 1 representing CC (for -141C Ins/Del) and CC/CT (for C957T). In each model, all predictors were entered as a block, after which genotype by treatment interaction terms were allowed to drop out in stepwise fashion ( $p$ to enter $=0.05, p$ to remove $=0.10$ ). To explore the longitudinal pattern of relapse by genotype, we examined time to failure (defined as 7 consecutive days of self-reported smoking) using Cox regression. Since a large number of people are left censored by not quitting on their target quit day (so they do not become part of the abstinent cohort), we also examined the left censoring dichotomy using logistic regression.

Genotype associations with changes in intermediate outcomes (withdrawal symptoms and side effects) were assessed using multivariate analysis of variance (MANOVA) to test genotype by time interactions. Genotype effects on post-treatment depression symptoms were assessed using linear regression models that controlled for baseline depression levels.

\section{Haplotype Analysis}

We estimated linkage disequilibrium (LD) between the DRD2 -141C Ins/Del and C957T SNPs using maximumlikelihood methods through the PWLD STATA routine. The two SNPs were in moderate LD $\left(D^{\prime}=0.70, p<0.001\right)$, 


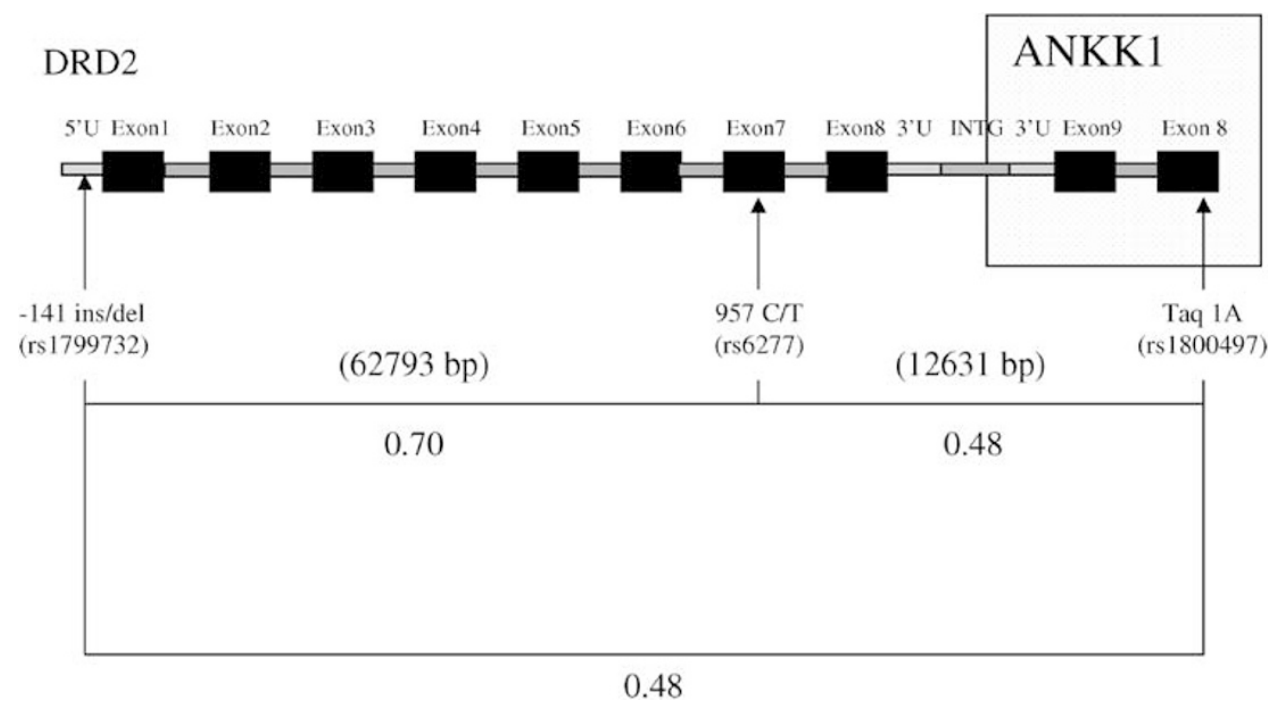

Figure 3 DRD2 gene with LD estimates $(n=772)$.

consistent with previous reports (Duan et al, 2003). The significance of disequilibrium was determined by estimating haplotype frequencies (with EM methods), in conjunction with a log-linear (contingency table) analysis, using the HAPIPF routine in STATA (Mander, 2001). The log-linear model was extended using HAPIPF for the analysis of haplotype by abstinence for the NRT study, and haplotypes by treatment by abstinence for the bupropion trial. Since HAPIPF identified one rare haplotype (N_T was $1.5 \%$ in the NRT study, and $1.4 \%$ in the bupropion study), the rare haplotype was eliminated and the remaining common haplotypes were analyzed for association with abstinence using ordinary chi-squared statistics. See Figure 3 for a diagram of the DRD2 gene and LD estimates for the pooled samples of trial participants.

\section{RESULTS}

\section{Study 1: Bupropion}

Descriptive data. Of the 414 eligible participants, $54 \%$ were female, $47 \%$ were college graduates, and the average age was $44.6(\mathrm{SD}=11.5)$ years. On average, participants smoked 22 cigarettes per day $(S D=9.3)$. The mean score on the Fagerström Test for Nicotine Dependence was 5.17 $(\mathrm{SD}=2.12)$. For the $-141 \mathrm{C}$ Ins/Del SNP, $348 \quad(84.1 \%)$ participants were homozygous for the $\mathrm{C}$ allele (CC), 59 $(14.2 \%)$ were heterozygous $(\mathrm{CN})$, and $7(1.7 \%)$ were homozygous for the $\mathrm{N}$ allele (NN). For the C957T SNP, 97 (23.4\%) participants were homozygous for the C allele (CC), $200(48.3 \%)$ were heterozygous (CT), and $117(28.3 \%)$ were homozygous for the T allele (TT). The C957T genotypes were in Hardy-Weinberg equilibrium $(p=0.6)$. The $-141 \mathrm{C}$ Ins/Del genotypes did not match Hardy-Weinberg equilibrium frequencies $(p=0.03)$. However, the $\chi^{2}$-value was heavily influenced by the expected number of deletion homozygotes (3.1 against 7 observed). The two SNPs were in moderate LD with each other $\left(D^{\prime}=0.69, p<0.0001\right)$.

In total, $191(46.1 \%)$ participants were randomized to the placebo condition, and $233(53.9 \%)$ to the bupropion condition. There were no significant differences in allele frequencies, genotypes, or covariates by treatment group. Younger age predicted loss to follow-up at EOT ( $t=1.78$, $p=0.07)$ and at 6 months $(t=2.28, p=0.02)$. There were no significant differences in genotypes, other covariates, or treatment assignment by follow-up status.

\section{Treatment Outcome}

At the end of the treatment (EOT) phase, 115 (27.8\%) participants were abstinent (32.7\% of bupropion group vs $22.0 \%$ of placebo group; $\chi^{2}(1)=5.92, p=0.02$ ). At the 6-month follow-up, $90(21.7 \%)$ were abstinent $(25.6 \%$ of bupropion group $v s 17.3 \%$ of placebo group; $\chi^{2}(1)=4.15$, $p=0.04)$. There were no significant main effects of $-141 \mathrm{C}$ Ins/Del genotype on abstinence at EOT $\left(\chi^{2}(2)=0.017\right.$, $p=0.99)$ or at 6 -month follow-up $\left(\chi^{2}(2)=0.23, p=0.89\right)$. The C957T SNP was also not significantly associated with abstinence at EOT $\left(\chi^{2}(2)=0.32, p=0.85\right)$ or at 6 -month follow-up $\left(\chi^{2}(2)=4.18, p=0.12\right)$.

The results of the logistic regression model of abstinence at EOT (Table 1) included a statistically significant interaction of $-141 \mathrm{C}$ Ins/Del genotype with treatment group $(\mathrm{OR}=4.99 ; 95 \% \mathrm{CI}=1.42,17.62 ; p=0.01)$. The form of the interaction can be seen in Figure 4. In the placebo condition, smokers with the CC genotype were less likely than those with CN/NN genotypes to be abstinent (19 vs $33 \%$, respectively); however, in the bupropion condition, those with the CC genotype were more likely than those with CN/NN genotypes to be abstinent (35 vs 20\%). The corresponding quit rates for the three-level genotype variable for the placebo group are $\mathrm{CC}=19 \%, \mathrm{CN}=29 \%$, and $\mathrm{NN}=50 \%(n=4)$ and for the bupropion group are $\mathrm{CC}=35 \%, \mathrm{CN}=22 \%$, and $\mathrm{NN}=0 \%(n=3)$. Females and smokers with higher nicotine dependence scores were significantly less likely to be abstinent at EOT than males and smokers with lower dependence scores; depression symptoms did not predict abstinence. In the model of abstinence at 6 months, the interaction of $-141 \mathrm{C}$ Ins/Del genotype with treatment approached significance $(\mathrm{OR}=3.32 ; \mathrm{CI}=0.86$, 
Table I Logistic Regression Models of Abstinence at EOT and 6-Month Follow-Up-Bupropion Trial (Study I)



${ }^{\mathrm{a} E O T}=$ end of treatment.



BUP $=$ bupropion

PLA $=$ placebo

$\mathrm{NS}=$ nicotine nasal spray

$\mathrm{TN}=$ transdermal nicotine

Figure 4 Abstinence rates by $D R D 2-|4| C$ Ins/Del genotype and treatment.

$12.80 ; p=0.08)$. The form of the interaction was similar to that seen at EOT. In contrast to the univariate results above, the main effect of C957T genotype was significant in the regression model $(\mathrm{OR}=0.59 ; \mathrm{CI}=0.35,0.99 ; p=0.048)$ (Table 1).

Application of survival methods revealed that the observed interaction between bupropion treatment and the $-141 \mathrm{C}$ genotype results entirely from an improved ability of subjects with the CC genotype (compared to the $\mathrm{CN} / \mathrm{NN}$ group) to quit on their target quit day in response to drug, an initial difference that carried through to 6 months. The results were confirmed by the logistic regression analysis of genotype by treatment effects on abstinence at the TQD (ie left censoring) ( $O R=4.63$; $\mathrm{CI}=1.39-15.48 ; p=0.01$ ), while Cox regression to estimate differences in relapse over time revealed no significant interaction $(\mathrm{HR}=0.59 ; \mathrm{CI}=0.26-1.39 ; p=0.23)$.

\section{Haplotype Analysis of Treatment Outcome}

There were four possible haplotype combinations arising from the DRD2 - 141C Ins/Del and C957T SNPs: C_C, C_T,
N_C, and N_T (position 1 represents the $-141 \mathrm{C}$ Ins/Del allele, and position 2 represents the C957T allele). HAPIPF estimated their respective haplotype frequencies to be $40.2,51.0,7.4$, and $1.4 \%$. Based on the results from the logistic regression, we tested for haplotype by treatment interaction overall results using the log-linear analysis in HAPIPF; this provided evidence of a significant association between haplotype/treatment combinations and point prevalence abstinence at EOT $(-2 \mathrm{LR}(7)=21.5$, $p=0.003)$, and at 6 months $(-2 \operatorname{LR}(7)=15.8, p=0.03)$. To avoid using the rare haplotype $\left(\mathrm{N}_{-} \mathrm{T}\right)$ in the analysis, we used the frequency estimates from the haplotype $\times$ treatment $v s$ abstinence analysis to perform contingency table analysis without the rare haplotype. As in the original log-linear analysis, results were significant at EOT $\left(\chi^{2}(5)=19.5, p=0.002\right)$, and at 6 months $\left(\chi^{2}(5)=14.4\right.$, $p=0.02)$. Consistent with the regression results described above, it appeared that haplotypes containing the -141 Ins C allele were associated with better response to bupropion treatment while those containing the -141 DelC allele were associated with better response to placebo (Table 2). 
Table 2 Estimated Haplotype Counts by Treatment in Bupropion Trial (Study I)

\begin{tabular}{|c|c|c|c|c|c|c|}
\hline \multirow[b]{2}{*}{ Haplotype } & \multicolumn{3}{|c|}{ Placebo } & \multicolumn{3}{|c|}{ Bupropion } \\
\hline & $\begin{array}{l}\text { Estimated } \\
\text { chromosome } \\
\text { sample }\end{array}$ & $\begin{array}{l}\text { Percent success } \\
\text { at EOT }\end{array}$ & $\begin{array}{l}\text { Percent success } \\
\text { at } 6 \text { months }\end{array}$ & $\begin{array}{l}\text { Estimated } \\
\text { chromosome } \\
\text { sample }\end{array}$ & $\begin{array}{l}\text { Percent success } \\
\text { at EOT }\end{array}$ & $\begin{array}{l}\text { Percent success } \\
\text { at } 6 \text { months }\end{array}$ \\
\hline$C_{-} C$ & 153.2 & 19.3 & 14.0 & 179.7 & 34.0 & 23.4 \\
\hline$C_{-} T$ & 188.8 & 21.4 & 18.3 & 233.3 & 33.9 & 28.7 \\
\hline
\end{tabular}

${ }^{\mathrm{a}} \mathrm{EOT}=$ end of treatment.

Haplotypes are written as - I4I Ins/Del C allele first, followed by the C957T allele.

$p=0.002$ for EOT, $p=0.02$ for 6 months

\section{Genotype Associations with Intermediate Outcomes}

The results of the MANOVAS revealed no evidence for genotype effects on changes in withdrawal symptoms or side effects (all $p$-values $>0.20$ ). The two genotypes were unrelated to changes in depression symptoms from baseline to EOT or baseline to 6-month follow-up (all p-values $>0.20$ ).

There was a nonsignificant difference in pill counts by $-141 \mathrm{C}$ genotype (CC group $=63.8 \pm 1.6$ vs $\mathrm{CN} / \mathrm{NN}$ group $=56.2 \pm 3.9 ; t=-1.89, p=0.06)$, but no difference by C957T genotype $(t=-0.54, p=0.58)$. Given the marginal association of genotype with pill counts, the logistic regression model was re-run including pill count as a covariate, and the results were unchanged. The odds ratio for the $-141 \mathrm{C}$ genotype by treatment interaction effect on abstinence, controlling for pill counts, was $4.70(p=0.018)$, compared with $\mathrm{OR}=4.99(p=0.012)$ in Table 1 .

\section{Study 2: NRT}

Descriptive data. Of the 368 eligible participants, $46 \%$ were female, $50 \%$ were college graduates, and the mean age was $47(\mathrm{SD}=11.1)$ years. On average, participants smoked 24 cigarettes per day $(\mathrm{SD}=9.3)$. The mean score for nicotine dependence was $5.58(\mathrm{SD}=2.22)$. For the $-141 \mathrm{C}$ Ins/Del SNP, $303(82.3 \%)$ participants were homozygous for the $\mathrm{C}$ allele (CC), 62 (16.8\%) were heterozygous (CN), and $3(0.8 \%)$ were homozygous for the $\mathrm{N}$ allele $(\mathrm{NN})$. For the C957T SNP, 115 (31.3\%) participants were homozygous for the T allele (TT), $182(49.5 \%)$ were heterozygous (CT), and 71 (19.3\%) were homozygous for the C allele (CC). Both loci were in Hardy-Weinberg equilibrium ( $p$-values $>0.8$ ).

Compared to participants in the bupropion trial, NRT trial participants were younger (44.6 vs $46.8 \mathrm{y}, p=0.006$ ), had lower levels of nicotine dependence (FTND score $=5.2$ $v s 5.6, p=0.008$ ), and were more likely to be female (54 vs $46 \%, p=0.04)$.

In total, $188(51.1 \%)$ participants were randomized to the NS treatment condition, and $180(48.9 \%)$ to the TN condition. There were no significant differences in allele frequencies, genotypes, or covariates by treatment group. Of the 368 eligible participants, $350(95.1 \%)$ provided data at EOT, and 353 (95.9\%) provided data at 6-month follow-up. There were no significant differences in genotypes, covariates, or treatment assignment by EOT completion status
( $p$-values $>0.10)$. Participants lost to follow-up at 6 months were more likely to have been allocated to the TN condition $\left(\chi^{2}(1)=3.73, p=0.05\right)$. There were no significant differences in genotypes or covariates by 6-month interview completion status.

\section{Treatment Outcome}

At the end of the NRT treatment phase (EOT), 124 (33.7\%) participants were abstinent (37.2\% of TN group $v s 30.3 \%$ of NS group; $\left.\chi^{2}(1)=1.96, p=0.16\right)$. At the 6 -month follow-up, $74(20.1 \%)$ were abstinent $(20.0 \%$ of TN group vs $20.2 \%$ of NS group; $\left.\chi^{2}(1)=0.01, p=0.96\right)$.

There was a significant association of $-141 \mathrm{C}$ Ins/Del genotype with abstinence at EOT $\left(\chi^{2}(1)=5.48, p=0.02\right)$. Overall, $46 \%$ of participants with $\mathrm{CN} / \mathrm{NN}$ genotypes were abstinent compared to $31 \%$ of those with the CC genotype (Figure 4); when the $\mathrm{CN}$ and $\mathrm{NN}$ groups are not combined, the quit rates at EOT are $31 \%$ for the CC group, $44 \%$ for the CN group, and $100 \%$ for the NN group $(n=3)$. This effect was no longer significant at 6-month follow-up $\left(\chi^{2}(1)=1.00, p=0.32\right)$. The C957T SNP was not significantly associated with abstinence at EOT $\left(\chi^{2}(2)=2.33\right.$, $p=0.31)$ or at 6 -month follow-up $\left(\chi^{2}(2)=0.12, p=0.94\right)$.

Results of the logistic regressions of abstinence at EOT and 6 months are shown in Table 3. Both DRD2 SNPs were significantly associated with abstinence at EOT, controlling for treatment group, sex, and nicotine dependence. For the $-141 \mathrm{C}$ Ins/Del locus, participants with CC genotypes were about one-half as likely to be abstinent at EOT as participants with $\mathrm{CN} / \mathrm{NN}$ genotypes $(\mathrm{OR}=0.44 ; \mathrm{CI}=0.25$, $0.79 ; p=0.006)$. For the C957T locus, participants with CT/CC genotypes were less than two-thirds as likely to be abstinent at EOT as participants with TT genotypes $(\mathrm{OR}=0.59 ; \mathrm{CI}=0.36,0.95 ; p=0.03)$. Genotype by treatment interactions were not significant. In the model of abstinence at 6 months, neither genotype contributed significantly and there were no genotype by treatment interactions. Depression symptoms did not predict abstinence at either time point ( $p$-values $>0.20)$.

The survival analysis suggested that the DRD2 -141C genotype effects on abstinence occur as a result of incremental differences in relapse/failure over time. Females failed more quickly than males $(\mathrm{HR}=1.57 ; \mathrm{CI}=1.19-2.08$; $p=0.002$ ), those with higher dependence failed more quickly (per unit FTND; $\mathrm{HR}=1.09 ; \quad \mathrm{CI}=1.02-1.16$; 
Table 3 Logistic Regression Models of Abstinence at EOT and 6-Month Follow-Up-NRT Trial (Study 2)

\begin{tabular}{|c|c|c|c|c|c|c|}
\hline \multirow[b]{2}{*}{ Predictor } & \multicolumn{3}{|c|}{ EOT $^{\mathrm{a}}$} & \multicolumn{3}{|c|}{6 months } \\
\hline & OR & $95 \% \mathrm{Cl}$ & $p$ & OR & $95 \% \mathrm{Cl}$ & $p$ \\
\hline Treatment $(N S=1)$ & 0.74 & $0.48,1.16$ & 0.19 & 1.06 & $0.63,1.77$ & 0.84 \\
\hline Nicotine dependence & 0.93 & $0.84,1.02$ & 0.14 & 0.90 & $0.81,1.02$ & 0.09 \\
\hline
\end{tabular}

${ }^{\mathrm{a}} \mathrm{EOT}=$ end of treatment.

Table 4 Estimated Haplotype Counts in NRT Trial (Study 2)

\begin{tabular}{lcccc}
\hline Follow-up & Haplotype & Failed & Abstained & \% Success \\
\hline $\mathrm{EOT}^{\mathrm{a}}$ & C_C & 196.04 & 70.93 & 26.57 \\
$\left(\chi_{(2 \mathrm{df})}^{2}=14.5\right.$, & & & & \\
$p<0.00 \mathrm{I})$ & C_T & 256.96 & 144.07 & 35.93 \\
& N_C & 27.96 & 29.07 & 50.98 \\
& & & & \\
& C_C & 214.84 & 52.14 & 19.53 \\
6 months & & & & \\
$\left(\chi_{(2 \mathrm{df})}^{2}=1.3\right.$, & C_T & 321.16 & 79.86 & 19.92 \\
$p=0.52)$ & N_C & 42.16 & 14.86 & 26.07 \\
& & & \\
& & & & \\
\hline
\end{tabular}

${ }^{\mathrm{a} E O T}=$ end of treatment.

Haplotypes are written as $-14 \mathrm{I}$ Ins/Del C allele first, followed by the C957T allele. $p=0.0008$ for EOT, $p=0.5$ at 6 -months.

$p=0.013)$, and those with the -141 CC genotype failed more quickly than those with $\mathrm{CN}$ or $\mathrm{NN}$ genotypes $(\mathrm{HR}=1.46 ; \mathrm{CI}=1.00-2.14 ; p=0.05)$.

\section{Haplotype Analysis of Treatment Outcome}

There were four possible haplotype combinations arising from the -141 C Ins/Del and C957T SNPs: C_C, C_T, N_C, and N_T (position 1 represents the $-141 \mathrm{C}$ Ins/Del allele, and position 2 represents the C957T allele). HAPIPF estimated their respective haploytpe frequencies to be $37.3,54.5,7.7$, and $1.5 \%$. Overall results from the log-linear analysis in HAPIPF provided evidence of an association between haplotype and abstinence at EOT $(-2 \operatorname{LR}(3)=$ 15.97, $p=0.0011)$, but no evidence of an association of haplotype with abstinence at 6 months $(-2 \mathrm{LR}(3)=4.30$, $p=0.23)$. To avoid using the rare haplotype $\left(\mathrm{N}_{-} \mathrm{T}\right)$ in the analysis, we used the frequency estimates from the haplotype and abstinence analysis to perform contingency table analysis without the rare haplotype. The results are shown in Table 4, and as in the original analysis, the association is significant at EOT $\left(\chi^{2}(2)=14.39, p=0.0008\right)$, but not at 6 months $\left(\chi^{2}(2)=1.24, p=0.5\right)$. Consistent with the regression analysis described above, at EOT, haplotypes containing the $-141 \mathrm{C}$ Ins/Del C allele were associated with a lower abstinence rate.

\section{Genotype Associations with Intermediate Outcomes}

The average change in withdrawal symptom scores from prequit to 1 -week postquit was $1.71 \pm 7.18$ (range $=-17-$ $30)$, and this did not differ by $-141 \mathrm{C}$ genotype $(t=1.6$, $p=0.11)$ or C957T genotype $(t=0.46, p=0.64)$. The average side effects score at 1 -week postquit was $26.95 \pm 5.15$ (range $=18-53$ ), and this measure also did not differ by $-141 \mathrm{C}$ genotype $(t=-0.92, p=0.36)$ or by C957T genotype $(t=-0.11, p=0.91)$.

The results of MANOVAS revealed no evidence for genotype effects on changes in withdrawal or side effects (all $p$-values $>0.20$ ). There were no significant effects of either genotype on changes in depression symptoms from baseline to EOT or baseline to 6-month follow-up (all $p$-values $>0.20$ ).

There was a significant difference in days per week of patch use by $-141 \mathrm{C}$ genotype (CC group $=6.4 \pm 1.3$ vs $\mathrm{CN} / \mathrm{NN}$ group $=6.9 \pm 0.17 ; t=4.41, \quad p=0.001)$ and by C957T genotype $(\mathrm{CC} / \mathrm{CT}$ group $=6.7 \pm 0.74$ vs $\mathrm{TT}$ group $=$ $6.1 \pm 1.79, t=-2.25, p=0.028)$. However, this is likely attributable to the fact that those participants who relapse to smoking are advised to discontinue patch use. The average number of nicotine spray doses was $9.59 \pm 7.95$, and this did not differ by $-141 \mathrm{C}$ genotype $(t=-0.52, p=0.60)$ or by C957T genotype $(t=0.20, p=0.84)$.

\section{Analysis of Population Stratification}

The STRUCTURE program (Pritchard et al, 2000) was used to test for the presence of ethnic admixture in the two study populations using 41 random biallelic SNPs (list of SNPs available upon request). We tested the hypothesis of two or more strata (ie ethnic subpopulations) within each study sample, and the program attempted to classify individuals as belonging to population or the other. The results included class probabilities for each individual. For example, an individual might be assigned a probability of 0.6 for belonging to one population, and 0.4 for belonging to the other. A stratified population would separate into a bimodal distribution of assignment probabilities, with values close to 0 or 1 . In both of our study samples, the values all were extremely close to 0.5 , indicating that the program could not identify separate strata within either of our study samples. As shown in Figure 5, probabilistic assignment was evenly split for all individuals. 


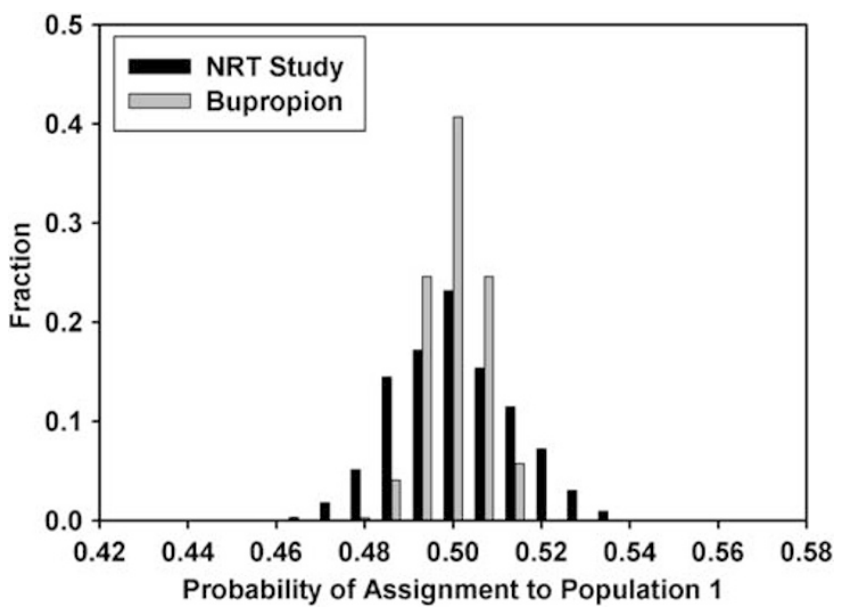

Figure 5 Assignment to population by the structure clustering program.

\section{DISCUSSION}

This study investigated the effects of two functional DRD2 SNPs on response to pharmacotherapy in two smoking cessation randomized clinical trials, a double-blind placebocontrolled trial of bupropion and an open label trial of TN $v s$ NS. The results of logistic regression and haplotype analyses revealed a significant bupropion treatment by genotype interaction at the EOT phase. Specifically, smokers homozygous for the InsC allele of the -141C Ins/Del SNP (ie CC) responded better to bupropion plus counseling than did smokers carrying the DelC allele (CN or NN genotypes). The difference in abstinence rates between genotype groups at EOT was also clinically significant (bupropion condition: 35 vs $20 \%$ for CC vs CN/NN, respectively; placebo condition: 19 vs 33\% for CC vs CN/NN, respectively). By contrast, smokers carrying one or more copies of the DelC allele at $-141 \mathrm{C}$ Ins/Del responded better to behavioral counseling alone (Study 1) and to NRT plus behavioral counseling (Study 2). A greater response to NRT was also observed among smokers who were homozygous for the $\mathrm{T}$ allele of the C957T SNP; however, this effect was significant only in the multivariable model when the effects of the $-141 \mathrm{C}$ Ins/ Del SNP and other covariates were considered. Haplotypes for the two SNPs were strongly predictive of treatment response in the bupropion trial ( $p=0.002$ and 0.02 for EOT and 6-months, respectively) and of abstinence at EOT in the NRT trial $(p=0.0008)$. However, these effects were attributable primarily to the association of abstinence with the DRD2 -141C Ins/Del SNP. Finally, it should be noted that genotype effects on response to treatment are markedly attenuated at 6-month follow-up. This may be expected for a true pharmacogenetic effect, as participants have been without medication for an extended period of time.

From a neurobiological perspective, these results may be interpreted as follows. Nicotine introduced into the ventral tegmental area (VTA) releases dopamine from nerve terminals in the nucleus accumbens (NAC) (Nisell et al, 1994), contributing to nicotine's rewarding effects ( $\mathrm{Di}$ Chiara and Imperato, 1988). Given that the -141C InsC allele results in higher transcriptional efficiency compared to the $\operatorname{Del}(\mathrm{N})$ allele (Arinami et al, 1997), individuals with the -141 Ins/Del CC genotype may have more D2 receptors available to bind dopamine, and smoking may be more rewarding in this group. Therefore, smokers with the CC genotype may have a greater liability to relapse than those who carry the DelC allele, consistent with the observation that smokers carrying the DelC allele have higher quit rates than those with the CC genotype following behavioral counseling alone (study 1) or NRT (study 2). In contrast to NRT, bupropion treatment (via inhibition of dopamine reuptake) may result in sustained elevations in extracellular dopamine; higher dopamine levels, in turn, may replace the hedonic (pleasurable) effects of smoking more effectively than NRT (Balfour, 2004). Thus, bupropion treatment may overcome the liability to relapse in individuals with the -141 C CC genotype because they have more D2 receptors to bind dopamine.

The mechanisms of genotype effect on abstinence and treatment response in these trials merit further investigation. There were no significant effects of either genotype on changes in side effects or withdrawal symptoms, suggesting that these effects on therapeutic response are attributable to other mechanisms. Survival analysis in the bupropion trial indicated that the effect of $-141 \mathrm{C}$ Ins/Del genotype on response to treatment occurs mainly at the TQD, an effect maintained through 6-month follow-up. This suggests the hypothesis that bupropion treatment (which occurs for 2 weeks prior to the TQD) may increase motivation to quit, rather than altering abstinence-related symptoms. In contrast, in the NRT trial, the main effect of genotype appeared to occur as a result of incremental differences in relapse over time, perhaps due to medication effects not measured in the current trial.

These findings extend previous pharmacogenetic investigations of bupropion and NRT focusing on the DRD2 Taq1A polymorphism. For example, greater beneficial effects of bupropion have been found among smokers with DRD2 Taq1 A2/A2 genotypes, as compared to those who carry the A1 allele (David et al, 2003). This finding is consistent with our results when one considers that a genotype associated with increased transcriptional efficiency in cell culture $(-141 \mathrm{C}$ Ins/Del CC) (Arinami et al, 1997) and a genotype correlated with normal ( $v s$ reduced) receptor density (DRD2 Taq1 A2A2) (Thompson et al, 1997) both predict a favorable response to bupropion relative to placebo. Since significant linkage disequilibrium between the $-141 \mathrm{C} \mathrm{Ins/Del}$ and the Taq1A is not observed (Gelernter et al, 1998), the effects of these two variants on therapeutic response are likely to be at least partially independent. To confirm this, the logistic regression analyses reported above were rerun including DRD2 Taq1A genotype as a covariate and the results were essentially unchanged (results not shown).

Data from the NRT trial are also compatible with previous reports that the DRD2 Taq1A A1 genotype is associated with a more favorable response to NRT (Johnstone et al, 2004; Yudkin et al, 2004). This genotype has been associated with reduced D2 receptor binding (Thompson et al, 1997). As described above, we found that genotypes containing the Del allele (CN/NN), associated with decreased transcriptional efficiency for the D2 receptor (Arinami et al, 1997) predict better response to NRT. Thus, in the case of these two independent polymorphisms, smokers with genotypes associated with altered receptor availability or function achieved higher quit rates with NRT. We also found that 
smokers homozygous for the C957T T allele, associated with decreased mRNA stability and translation (Duan et al, 2003), exhibited a better response to NRT. Taken together, the findings from the bupropion and NRT trials are compatible with the premise that genotypes associated with reduced D2 receptor availability or function predict better outcomes with NRT, while those associated with normal receptor expression or function predict a better response to bupropion.

The present study has both strengths and limitations. The strengths include the collection of DNA samples from all study participants, rather than retrospective DNA collection that may result in bias. An additional strength is the empirical evaluation of the presence of population stratification, the results of which provided no evidence for population substructure that could bias the study results. One limitation of the study is that we did not collect posttreatment samples to determine levels of bupropion to verify self-reported medication compliance. A second limitation is the restricted ethnic background of participants, all of whom were of European origin. Thirdly, although the sample sizes of 414 and 368 afforded sufficient power to detect a clinically meaningful genotype by treatment interaction, it was not possible to test for genegene interactions because of the small cells generated for participants with the less common alleles for both genotypes. Previous evidence for interacting effects of the DRD2 Taq1A and dopamine transporter (SLC6A3) variants on smoking cessation suggests that testing for epistatic effects is important (Lerman et al, 2003).

Larger scale pharmacogenetic trials confirming the findings of the present study in different ethnic groups may allow for genotype-based selection of pharmacotherapy for nicotine dependence. A recent survey of primary care physicians suggests a high level of interest in adoption of genetic testing to tailor smoking cessation treatment (Shields et al, in press). In parallel to the pharmacogenetics research effort in smoking cessation treatment, there will need to be an investigation of the psychosocial, ethical, and health policy issues raised by the translation of findings to clinical practice (Shields et al, 2004, 2005).

\section{ACKNOWLEDGEMENTS}

We acknowledge the helpful contributions of TTURC staff members to the implementation of this study, especially Angela Pinto and Susan Ware. Thanks also to Maryanne Foster for assistance with manuscript preparation. We also thank Dr Peter Shields for helpful comments on the initial study design and are grateful to Dr David Balfour who provided insightful comments on a draft of this manuscript. Study medication for the bupropion trial was provided by GlaxoSmithKline. Nicotine nasal spray (Nicotrol) was provided by Pharmacia and Upjohn, Helsingborg, Sweden. This research was supported by grants from the National Cancer Institute and National Institutes of Drug Abuse, P50CA/DA84718 and RO1CA 63562 (CL) and a grant from the Pennsylvania Department of Health. The Department of Health specifically disclaims responsibility for any analyses, interpretations, or conclusions. Financial Disclosure: $\mathrm{Dr}$ Berrettini acts as a consultant to GlaxoSmithKline.

\section{REFERENCES}

Arinami T, Gao M, Hamaguchi H, Toru M (1997). A functional polymorphism in the promoter region of the dopamine D2 receptor gene is associated with schizophrenia. Hum Mol Genet 6: $577-582$.

Ascher J, Cole J, Colin J (1995). Bupropion: A review of its mechanism of antidepressant activity. J Clin Psychiatry 56: 395-401.

Balfour DJ (2004). The neurobiology of tobacco dependence: a preclinical perspective on the role of the dopamine projections to the nucleus. Nicotine Tob Res 6: 899-912.

Bierut L, Rice J, Edenberg H, Goate A, Foroud T, Cloninger C (2000). Family-based study of the association of the dopamine D2 receptor gene (DRD2) with habitual smoking. Am J Med Genet 90: 299-302.

Breen G, Brown J, Maude S, Fox H, Collier D, Li T et al (1999). 141 $\mathrm{C}$ del/ins polymorphism of the dopamine receptor 2 gene is associated with schizophrenia in a British population. Am J Med Genet 88: 407-410.

Brown R, Burgess E, Sales S, Whiteley J (1998). Reliability and validity of a smoking timeline follow-back interview. Psychol Addictive Behav 12: 101-112.

Cinciripini P, Wetter D, Tomlinson G, Tsoh J, De Moor C, Cinciripini L et al (2004). The effects of the DRD2 polymorphism on smoking cessation and negative affect: Evidence for a pharmacogenetic effect on mood. Nicotine Tob Res 6: 229-240.

Dale LC, Glover ED, Sachs DP, Schroeder DR, Offord KP, Croghan IT et al (2001). Bupropion for smoking cessation: predictors of successful outcome. Chest 119: 1357-1364.

David SP, Niaura R, Papandonatos GD, Shadel WG, Burkholder GJ, Britt DM et al (2003). Does the DRD2-Taq1 A polymorphism influence treatment response to bupropion hydrochloride for reduction of the nicotine withdrawal syndrome? Nicotine Tob Res 5: 935-942.

Di Chiara G, Imperato A (1988). Drugs abused by humans preferentially increase synaptic dopamine concentrations in the mesolimbic system of freely moving rats. Proc Natl Acad Sci USA 85: $5274-5278$.

Duan J, Wainwright MS, Comeron JM, Saitou N, Sanders AR, Gelernter J et al (2003). Synonymous mutations in the human dopamine receptor D2 (DRD2) affect mRNA stability and synthesis of the receptor. Hum Mol Genet 12: 205-216.

Evans WE, Relling MV (2004). Moving towards individualized medicine with pharmacogenomics. Nature 429: 464-468.

Feyerabend C, Russell MA (1990). A rapid gas-liquid chromatographic method for the determination of cotinine and nicotine in biological fluids. J Pharm Pharmacol 42: 450-452.

Fiore MC, Kenford SL, Jorenby DE, Wetter DW, Smith SS, Baker TB (1994). Two studies of the clinical effectiveness of the nicotine patch with different counseling treatments. Chest 105: 524-533.

Gelernter J, Kranzler H, Cubells JF, Ichinose H, Nagatsu T (1998). DRD2 allele frequencies and linkage disequilibria, including the -141 CIns/Del promoter polymorphism, in European-American, African-American, and Japanese subjects. Genomics 51: 21-26.

Gilbert D, McClernon J, Rabinovich N, Sugai C, Plath L, Asgaard G et al (2004). Effects of quitting smoking on EEG activation and attention last for more than 31 days and are more severe with stress, dependence, DRD2 A1 allele, and depressive traits. Nicotine Tob Res 6: 249-267.

Heatherton TF, Kozlowski LT, Frecker RC, Fagerstrom KO (1991). The Fagerstrom test for nicotine dependence: a revision of the Fagerstrom Tolerance Questionnaire. $\mathrm{Br} J$ Addict 86: 1119-1127.

Hughes J, Hatsukami D, Pickens RW, Krahn D, Malin S, Luknic A (1984). Effect of nicotine on the tobacco withdrawal syndrome. Psychopharmacology (Berl) 83: 82-87. 
Hughes JR, Keely JP, Niaura R, Ossip-Klein DJ, Richmond RL, Swan GE (2003). Measure of abstinence in clinical trials: issues and recommendations. Nicotine Tob Res 5: 13-25.

Hurt RD, Sachs DP, Glover ED, Offord KP, Johnston JA, Dale LC et al (1997). A comparison of sustained-release bupropion and placebo for smoking cessation. N Engl J Med 337: 1195-1202.

Johnstone EC, Yudkin PL, Hey K, Roberts SJ, Welch SJ, Murphy MF et al (2004). Genetic variation in dopaminergic pathways and short-term effectiveness of the nicotine patch. Pharmacogenetics 14: $83-90$.

Jorenby DE, Leischow SJ, Nides MA, Rennard SI, Johnston JA, Hughes AR et al (1999). A controlled trial of sustained-release bupropion, a nicotine patch, or both for smoking cessation. N Engl J Med 340: 685-691.

Lerman C, Kaufmann V, Rukstalis M, Patterson F, Perkins K, Audrain-McGovern J et al (2004a). Individualizing nicotine replacement therapy for the treatment of tobacco dependence: a randomized trial. Ann Intern Med 140: 426-433.

Lerman C, Niaura R (2002). Applying genetic approaches to the treatment of nicotine dependence. Oncogene 21: 7412-7420.

Lerman C, Patterson F, Berrettini W (2005). Treating tobacco dependence: state of the science and new directions. J Clin Oncol 23: 311-323.

Lerman C, Roth D, Kaufmann V, Audrain J, Hawk L, Liu A et al (2002). Mediating mechanisms for the impact of bupropion in smoking cessation treatment. Drug Alcohol Depend 67: 219-223.

Lerman C, Shields PG, Wileyto EP, Audrain J, Hawk Jr LH, Pinto A et al (2003). Effects of dopamine transporter and receptor polymorphisms on smoking cessation in a bupropion clinical trial. Health Psychol 22: 541-548.

Lerman C, Wileyto EP, Patterson F, Rukstalis M, AudrainMcGovern J, Restine S et al (2004b). The functional mu opioid receptor (OPRM1) Asn40Asp variant predicts short-term response to nicotine replacement therapy in a clinical trial. Pharmacogenom J 4: 184-192.

Mander AP (2001). Haplotype analysis in population-based association studies. STATA J 1: 58-75.

Neville MJ, Johnstone EC, Walton RT (2004). Identification and characterization of ANKK1: a novel kinase gene closely linked to DRD2 on chromosome band 11q23.1. Hum Mutat 23: 540-545.

Nisell M, Nomikos GG, Svensson TH (1994). Infusion of nicotine in the ventral tegmental area or the nucleus accumbens of the rat differentially affects accumbal dopamine release. Pharmacol Toxicol 75: 348-352.

Noble EP, Blum K, Ritchie T, Montgomery A, Sheridan PJ (1991). Allelic association of the D2 dopamine receptor gene with receptor-binding characteristics in alcoholism. Arch Gen Psychiatry 48: 648-654.
Piasecki T, Niaura R, Shadel W, Abrams D, Goldstein M, Fiore M et al (2000). Smoking withdrawal dynamics in unaided quitters. J Abnorm psychol 109: 74-86.

Pontieri F, Tanda G, Orzi F, Di Chiara G (1996). Effects of nicotine on the nucleus accumbens and similarity to those of addictive drugs. Nature 382: 255-257.

Pritchard JK, Stephens M, Donnelly P (2000). Inference of population structure using multilocus genotype data. Genetics 155: 945-959.

Radloff LS (1977). The CES-D Scale: a self-report depression scale for research in the general population. Appl Psychol Meas 1: 385-401.

Ritchie T, Noble EP (1996). [3H]naloxone binding in the human brain: alcoholism and the TaqI A D2 dopamine receptor polymorphism. Brain Res 718: 193-197.

Roses AD (2000). Pharmacogenetics and the practice of medicine. Nature 405: 857-865.

Sanchez C, Hyttel J (1999). Comparison of the effects of antidepressants and their metabolites on reuptake of biogenic amines and on receptor binding. Cell Mol Neurobiol 19: 467-489.

Shields A, Blumenthal D, Weiss K, Comstock C, Currivan D, Lerman C et al (2005). Barriers to translating genetic research on smoking into clinical practice: results from a National Survey of Primary Care Physicians. J Gen Intern Med 20: 131-138.

Shields A, Fortun M, Hammonds E, King P, Lerman C, Rapp R et al (2005). The use of race variables in genetic studies of complex traits and the goal of reducing health disparities: a transdisciplinary perspective. Am Psychol 60: 77-103.

Shields A, Lerman C, Sullivan P (2004). Translating emerging research on the genetics of smoking into clinical practice: Ethical and social considerations. Nicotine Tob Res 6: 675-688.

Spitz M, Shi H, Yang F, Hudmon K, Jiang H, Chanberlain R (1998). Case-control study of the D2 dopamine receptor gene and smoking status in lung cancer patients. J Natl Cancer Inst 90: 358-363.

SRNT Subcommittee on Biochemical Verification (2002). Biochemical verification of tobacco use and cessation. Nicotine Tob Res 4: 149-159.

Thompson J, Thomas N, Singleton A, Piggott M, Lloyd S, Perry EK et al (1997). D2 dopamine receptor gene (DRD2) Taq1 A polymorphism: reduced dopamine D2 receptor binding in the human striatum associated with the A1 allele. Pharmacogenetics 7: 479-484.

Transdermal Nicotine Study Group (1991). Transdermal nicotine for smoking cessation: six-month results from two multicenter controlled trials. J Am Med Assoc 266: 3133-3138.

Yudkin P, Munafo M, Hey K, Roberts S, Welch S, Johnstone E et al (2004). Effectiveness of nicotine patches in relation to genotype in women versus men: randomised controlled trial. $\mathrm{Br}$ Med J 328 : 989-990. 